\title{
Actividad ovárica del pampanito, Stromateus stellatus en el litoral de la Región del Biobío, Chile
}

\author{
Ovarian activity in Pacific butterfish, Stromateus stellatus in the coast off Biobío Region, Chile
}

\section{Claudio Carocca ${ }^{1}$ y Javier Chong ${ }^{1}$}

${ }^{1}$ Facultad de Ciencias, Universidad Católica de la Santísima Concepción, Caupolicán 491, Casilla 297, Concepción, Chile. jvchong@ucsc.cl

\begin{abstract}
A study on the reproductive biology of the Pacific butterfish, Stromateus stellatus, using samples from the coast off Biobío Region (Chile), was carried out. Samples were obtained from fishing vessels during austral spring and summer months. Microscopic analysis shows the presence of oocytes at different developmental stages in the same ovaries, a feature consistent with asynchronous spawning. The frequency of maturity stages shows dominance of mature specimens (90\%) in October, previtellogenic in December (33\%), and an increase of vitellogenic (73\%) and mature (21\%) specimens in J anuary. This suggests spawning events in November and February, and a reduced reproductive activity in December. The average size at first maturity was $21.1 \mathrm{~cm} \mathrm{TL}$. Partial fecundity varied from $8,340(\mathrm{TL}=20.6 \mathrm{~cm})$ to $36,210(\mathrm{TL}=28.1 \mathrm{~cm})$ oocytes. The relationship between partial fecundity and size is represented by $\mathrm{Fp}=14.832 \mathrm{TL}^{2.258}\left(\mathrm{R}^{2}=0.3043\right)$.
\end{abstract}

Key words: Reproduction, asynchronous spawning, fecundity, sexual maturity

\section{INTRODUCCIÓN}

Stromateus stellatus, denominado pampanito, es un pequeño pez pelágico, único representante de la familia Stromateidae en las costas de Chile, que se distribuye desde Perú hasta la zona austral de Chile (Chirichigno \& Cornejo 2001). Está asociado a poblaciones de sardinas y anchovetas, alimentándose de larvas de estas especies (Leible \& Alveal 1981) y constituye parte de la fauna concurrente en las capturas de recursos como Engraulis ringens (Jenyns, 1842), Strangomera bentincki (Norman, 1936), Merluccius gayi gayi (Guichenot, 1848) y Trachurus murphyi (Nichols, 1920). El pampanito presenta toxinas en la piel y musculatura que provoca hemorragias intestinales o diarreas, por lo que no es apto para el consumo humano (Sato et al. 2002), pero se utiliza en la elaboración de harina de pescado. También se conoce que las poblaciones de $S$. stellatus son abundantes, aunque se ignora el efecto de la explotación y sólo se cuenta con información parcial de desembarques, de 1.579 ton 2003 a 7.520 ton en 2006 (SERNAPESCA 2006). En la costa centro-sur de Chile, los fuertes eventos de surgencia que se desarrollan en la época estival, aumentan la productividad biológica, lo que permite la maduración, desove y sustentabilidad de algunas poblaciones de especies pelágicas (Oliva et al. 1986, Cubillos et al. 2001). En este contexto, E. ringens y $S$. bentincki poseen periodos reproductivos extensos donde el desove se produce a través de tandas sucesivas, evitando arriesgar todo el material gonádico en una sola puesta (Vizziano \& Borois 1990), comportamiento que también podría presentar $S$. stellatus que cohabita con tales especies.

Los escasos antecedentes reproductivos para $S$. stellatus han sido informados para la costa peruana (Elliott et al. 2000) y para Stromateus brasiliensis Fowler de la costa Atlántica por Perrotta et al. (2006).

La ausencia de antecedentes reproductivos en S. stellatus frente al litoral chileno y la oportunidad de contar con muestras durante el periodo estival, permitió investigar la biología reproductiva, relacionada con el tipo y época de desove, fecundidad y longitud de madurez sexual en hembras de esta especie.

\section{Material y MÉtodos}

Se obtuvieron ejemplares de pampanito, Stromateus stellatus (fauna acompañante) desde embarcaciones industriales de pesca de arrastre de merluza común que operaron al suroeste de Isla Mocha, Chile central ( $38^{\circ} 23^{\prime} \mathrm{S}$, $73^{\circ} 52^{\prime} \mathrm{W}$ ), durante los meses de octubre y diciembre de 2005 y enero de 2006. Los especímenes se obtuvieron a bordo de las embarcaciones en muestras aleatorias obtenidas en cada lance de pesca. Posteriormente en laboratorio, a cada ejemplar hembra se le registró la longitud 
total (LT, cm), peso total (PT, g), peso gonadal (PG, g) y se evaluó el estado de madurez sexual según la escala macroscópica de seis estados según Murúa et al. (2003). Una submuestra de 230 hembras se examinaron microscopicamente, para lo cual se extrajeron las gónadas, fijándolas en formalina al 10\%, y luego sometidas a técnicas histológicas convencionales, para obtener cortes de $6 \mu \mathrm{m}$ que fueron teñidos con hematoxilina-eosina (Oliva et al. 1986).

El análisis para establecer la condición reproductiva estival en hembras de pampanito consideró:

a. Examen microscópico, los ovarios fueron clasificados en una escala de 5 estados de madurez (Oliva et al. 1986): inactivo o en reposo (I), previtelogénico (PV), vitelogénico (V), maduro (M) y desove (D) (presencia de folículos post-ovulatorios).

b. Indice Gonadosomático (IGS), indica la mayor actividad reproductiva cuando alcanza valores máximos en su ciclo:

$$
I G S=\frac{P \cdot \text { gónada }}{P \cdot \text { pez }-P \cdot \text { gónada }} 100
$$

La variación temporal de los valores de IGS, se compararon mediante la aplicación de un análisis de varianza y un test post hoc de Tukey (Zar 1984).

c. Examen macroscópico, se estableció sobre la base de una escala de madurez de seis estados: inmaduro, madurando, maduro, desove, desovado y reposo (Murúa et al. 2003).

d. Fecundidad parcial, fue estimada mediante el conteo de los ovocitos correspondientes a la moda de tamaño más avanzada (MOMA), que es aquella que será desovada en primer lugar durante la estación. Esta fue separada de otras menores a través de un sistema de tamices que fluctuó entre 100 y $500 \mu \mathrm{m}$, separados cada $50 \mu \mathrm{m}$ siguiendo la metodología de Herrera \& Claramunt (1990). La fecundidad se calculó en 45 hembras maduras, capturadas en octubre donde el IGS fue mayor y hubo ausencia de folículos post-ovulatorios (FPO). El recuento correspondió a ovocitos maduros de diámetros $\geq 500 \mu \mathrm{m}$, utilizado por otros autores (Hunter \& Leong 1981, Chong et al. 1991, Claramunt \& Herrera 1992).

En la determinación de la fecundidad parcial se utilizó la expresión de Oliva et al. (1986):

$$
F=\frac{1}{n} \sum_{i=1}^{n}\left(\frac{H_{i}}{W S_{i}}\right) * W_{o}
$$

Donde:

$\mathrm{H}_{i} \quad$ = número de ovocitos en la submuestra $i$

$\mathrm{W}_{0}=$ peso de la gónada $(\mathrm{g})$

$\mathrm{WS}_{i}=$ peso de la submuestra $i(\mathrm{~g})$

Los valores de fecundidad parcial se relacionaron con la longitud total (LT) y el peso total (PT) de acuerdo con la función que entregara el mejor ajuste (potencial, lineal, logarítmica).

e. Longitud de primera madurez sexual, corresponde a la longitud de un ejemplar que se reproduce por primera vez y desde el punto de vista poblacional, se define como la longitud en que el $50 \%$ de los individuos de una población están maduros. El grado de madurez se estableció mediante el examen microscópico considerándose como inmaduro los estados inactivo y previtelogénico. En su determinación se utilizó la ecuación logística (Hosmer \& Lemeshow 1989, Roa et al. 1999):

$$
P(l)=\frac{1}{1+e^{\left(\beta 1+\beta 2^{*} l\right)}}
$$

Donde: $P_{(l)}$ corresponde a la proporción de hembras maduras a la longitud total (l) y $\beta_{1}+\beta_{2}$ son constantes del modelo.

Tabla 1. Rangos de longitud, promedio y desviación estándar mensual de Stromateus stellatus y valores medios de IGS / Monthly length range, mean and standard deviation of Stromateus stellatus and mean of IGS values

\begin{tabular}{lcccccc}
\hline Meses & Rango LT & Promedio & D. estándar & Prom. IGS & D. estándar & Muestras \\
\hline Octubre-05 & $19,7-28,4$ & 24,46 & 1,99 & 4,75 & 1,75 & 230 \\
Dic-05 & $17,5-28,1$ & 22,29 & 2,42 & 1,94 & 0,93 & 175 \\
Enero-06 & $18,0-28,4$ & 22,90 & 2,71 & 2,26 & 1,44 & 30 \\
\hline
\end{tabular}




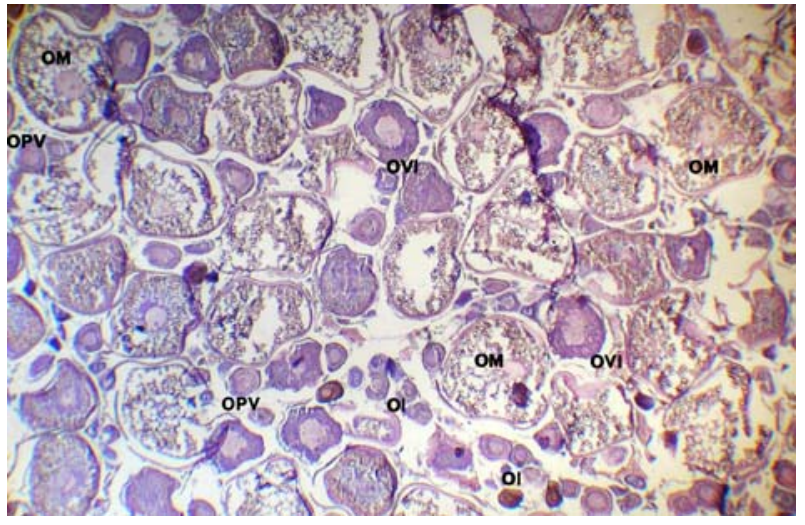

Figura 1. Ovario maduro de S. stellatus con presencia de ovocitos maduro (OM), vitelogénicos (OVI), previtelogénicos (OPV) e inactivos (OI) / Mature ovary of $S$. stellatus with mature (OM), vitelogenic (OVI), previtelogenic (OPV) and inactive oocyte (OI)

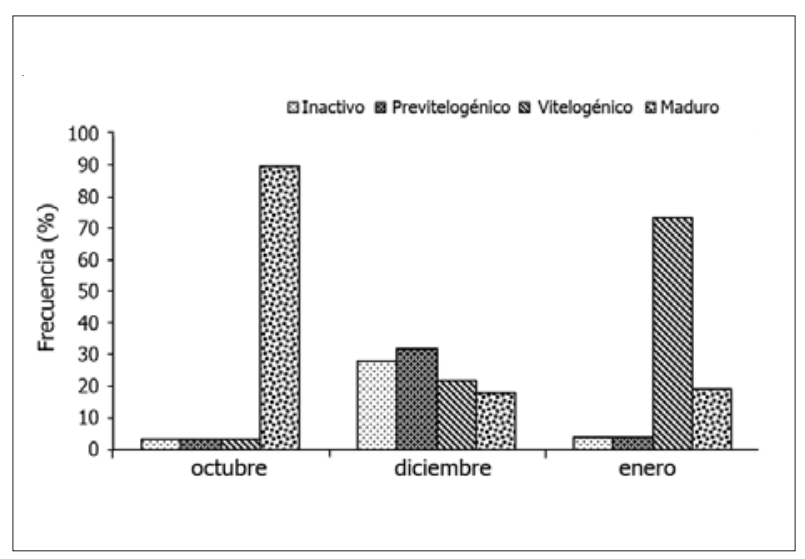

Figura 2. Frecuencia mensual (\%) de estados microscópicos de madurez sexual en hembras de S. stellatus / Monthly frequency (\%) of microscopic maturity stages in females of S. stellatus

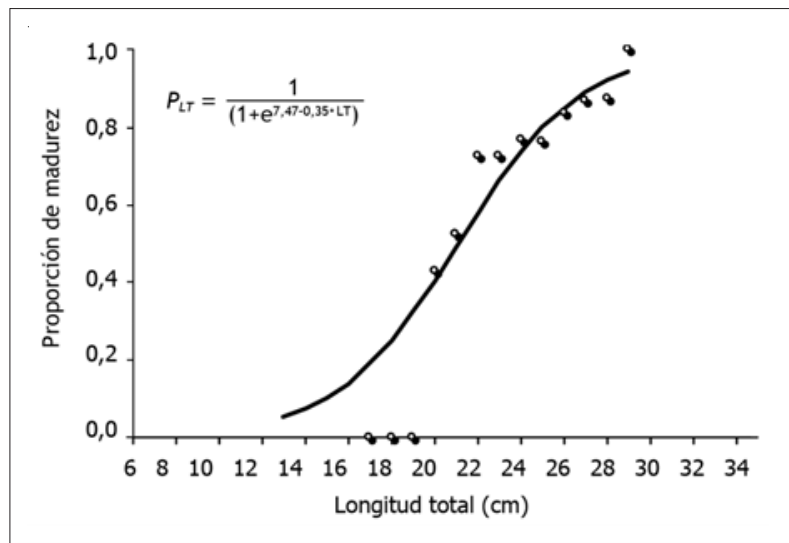

Figura 3. Longitud de primera madurez sexual $\left(L_{50}=21,13 \mathrm{~cm}\right)$ para hembras de S. stellatus / Length of first sexual maturity $\left(L_{50}=\right.$ $21.13 \mathrm{~cm}$ ) for females of $\mathrm{S}$. stellatus

\section{Resultados}

Se analizaron 444 hembras de $S$. stellatus con longitudes entre 17 y $29 \mathrm{~cm}$ LT (Tabla 1), cuya relación con el peso está representada por la ecuación PT $=0,0235$ $\mathrm{LT}^{2,773}\left(\mathrm{R}^{2}=0,801\right)$.

El examen microscópico de 230 hembras permitió determinar que la especie posee un desove de tipo asincrónico, al presentar ovocitos en distintos estado de desarrollo en un mismo ovario maduro (Fig. 1). La frecuencia de los estados de madurez muestra un predominio de ejemplares maduros (90\%) en octubre, una disminución de los estados maduro (18\%) y vitelogénico (21\%) en diciembre y una dominancia de los estados vitelogénico (73\%) y maduro (21\%) en enero (Fig. 2). Esta situación podría indicar un periodo prolongado de puesta con épocas de mayor actividad ovárica en noviembre y febrero, comportamiento típico de un desovante parcial.

El análisis del IGS (Tabla 1) muestra el mayor valor promedio en el mes de octubre $(4,75)$, que disminuyó hacia diciembre $(1,94)$ y se incrementó levemente en enero $(2,26)$. Estas fluctuaciones son concordantes con el examen microscópico. No obstante, se encontró diferencias en los valores del IGS, resultando el mes de octubre significativamente distinto de diciembre y enero $(P<0,05)$.

La frecuencia de los estados de madurez macroscópicos, muestra una predominancia de hembras maduras (54\%) en octubre y de hembras madurando (60\%) en diciembre; en cambio en enero hay igual porcentaje (47\%) de hembras madurando y maduras. En octubre también se determinaron hembras en desove (18\%), reposo y desovada, lo que difiere con el examen microscópico que no registró tales estados, indicando la importancia del examen histológico y la reserva al considerar el examen macroscópico que, no obstante son necesarios por el alto costo de los primeros.

La longitud de primera madurez sexual $\left(L_{50}\right)$ estimada por el método de la función logística entregó una LT = 21,13 cm para las hembras de $S$. stellatus, siendo representada por la siguiente ecuación (Fig. 3):

$$
L_{(50)}=\frac{1}{1+\exp ^{(7,47-0,39 * L T)}} .
$$

Sin embargo, el individuo maduro más pequeño fue de $20 \mathrm{~cm}$ y el inmaduro de mayor longitud de $25 \mathrm{~cm}$.

La fecundidad parcial determinada en 45 hembras de $S$. stellatus fluctuó entre 8.340 y 36.210 ovocitos para 


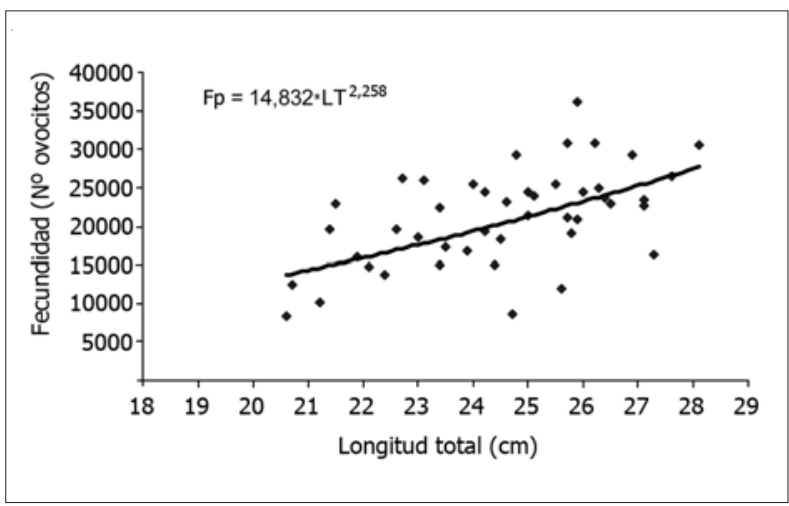

Figura 4. Relación entre la longitud total y la fecundidad parcial en hembras de S. stellatus / Relationship between total length and partial fecundity in female of $\mathrm{S}$. stellatus

longitudes de 20,6 y 28,1 cm LT, respectivamente con un promedio de $21.226 \pm 929$ ovocitos. La relación entre la longitud total y fecundidad parcial entregó el mejor ajuste mediante la relación potencial: $\mathrm{Fp}=14,832 * \mathrm{LT}^{2,25}\left(\mathrm{R}^{2}=\right.$ 0,3043; $P<0,01$ ) (Fig. 4). De igual forma, la relación entre el peso total y la fecundidad parcial también se ajustó mejor por la siguiente función potencial, $\mathrm{Fp}=325,991 * \mathrm{PT}$ $0,8048\left(\mathrm{R}^{2}=0,3504 ; P<0,01\right)$.

\section{Discusión Y CONCLUSIONES}

El análisis histológico determinó que $S$. stellatus es un desovante parcial o múltiple por presentar simultáneamente ovocitos en distintas fases de desarrollo en un mismo ovario maduro durante el periodo de estudio (Hunter \& Goldberg 1980, Chong et al. 1991, Murúa \& Saborido-Rey 2003). Este tipo de desove, caracterizado por un prolongado periodo de puesta con épocas de máxima intensidad permite enfrentar variaciones ambientales sin arriesgar todo el potencial reproductivo en una puesta (Vizziano \& Borois 1990). Esta modalidad reproductiva la presentan varias especies que conviven con el pampanito y que habitan estas latitudes asociadas a áreas de surgencias, como ha sido señalado por Cubillos et al. (1999) para E. ringens y S. bentincki, Chong \& González (1995) para Paralichthys microps y Chong \& González (2009) para Genypterus chilensis.

La frecuencia de los estados de madurez microscópicos muestra una fuerte actividad gonádica en primavera y verano con probables eventos de desove en noviembre y febrero-marzo con una disminución en diciembre. Este comportamiento reproductivo, consistente en dos periodos de puesta, uno principal en primavera y uno secundario al final de verano separados por un periodo de menor actividad al inicio del verano, ha sido previamente señalado para otras especies que interactúan con $S$. stellatus en la zona pesquera centro sur del país, como es el caso de P. microps (Chong \& González 1995), E. ringens, S. bentincki (Cubillos et al. 1999), Merluccius gayi (Balbontín \& Fischer 1981) y G. chilensis (Chong \& González 2009). La coincidencia espacio-temporal del proceso de madurez y desove de estas especies, estaría asociado a factores bióticos y abióticos favorables que inducen una alta productividad que desencadenaría el desove (Hislop et al. 1978, Hunter \& Leong 1981, Ware 1984, Brooks et al. 1997, Alheit \& Bernal 1993). Situación muy distinta fue informada para $S$. stellatus frente al litoral peruano, donde la madurez sexual se inicia en mayo con un máximo desove en agosto y septiembre (Elliott et al. 2000), diferencia que estaría asociada a distintas condiciones del ambiente. También, en forma puntual Perrotta et al. (2006) señalan que el pampanito (S. brasiliensis) frente a las costas argentinas $\left(34^{\circ}-38^{\circ} \mathrm{S}\right)$, presenta una madurez avanzada durante julio.

La longitud de primera madurez sexual estimada de 21,13 cm por medio de la función logística, muestra un buen ajuste, pues se contó con grupos de longitudes inferiores y superiores con $100 \%$ de inmadurez y madurez, respectivamente. Sin embargo, para la costa peruana Elliott et al. (2000) estiman una longitud de $15,5 \mathrm{~cm}$, pero con longitudes máximas menores $(22 \mathrm{~cm})$, lo que indicaría una madurez precoz en relación con la población en aguas chilenas. Para S. brasiliensis la longitud media de madurez es de 23,1 cm, para longitudes entre 6 a $40 \mathrm{~cm}$ (Perrotta et al. 2006).

En relación con la fecundidad parcial, los valores determinados podrían estar sobreestimados, ya que se basaron en conteos de ovocitos maduros, que podrían experimentar un proceso de atresia en su posterior desarrollo, por lo cual sería recomendable la utilización de ovocitos hidratados que son los efectivamente desovados y de mayor diámetro, que a la vez facilitaría su contaje.

Futuros trabajos sobre biología reproductiva del $S$. stellatus deberían considerar la obtención de muestras mensuales durante un lapso más extenso y continuo de tiempo, principalmente primavera, verano y otoño, lo que permitiría completar y corroborar lo registrado en esta comunicación.

\section{Agradecimientos}

Nuestros agradecimientos a la Dirección de la Facultad de Ciencias, Universidad Católica de la Santísima Concepción 
que contribuyó a financiar el presente trabajo y a dos revisores anónimos por sus sugerencias y observaciones al manuscrito.

\section{LITERATURA CITADA}

Alheit J \& PA Bernal. 1993. Effect of physical and biological changes on the biomasa yield of the Humboldt Current System. En: Sherman K, LM Alexander \& BD Gold (eds). Large marine ecosystems, pp. 53-68. American Association for the Advancement of Science Press, Washington.

Balbontín F \& W Fischer. 1981. Ciclo sexual y fecundidad de la merluza, Merluccius gayi gayi, en la costa de Chile. Revista de Biología Marina 17(3): 285-234.

Brooks S, CR Tyler \& JP Sumpter. 1997. Egg quality in fish: what makes a good egg? Reviews in Fish Biology and Fisheries 7: 387-416.

Chirichigno N \& RM Cornejo. 2001. Catálogo comentado de los peces marinos del Perú, 314 pp. Publicación Especial, Instituto del Mar del Perú, Callao.

Chong J \& P González. 1995. Ciclo reproductivo del lenguado de ojos chicos, Paralichthys microps (Günther 1881) (Pleuronectiformes, Paralichthydae) frente al litoral de Concepción, Chile. Biología Pesquera 24: 39-50.

Chong J \& P González. 2009. Ciclo reproductivo y talla media de madurez del congrio colorado, Genypterus chilensis (Guichenot, 1881) en el litoral de Talcahuano, Chile. Revista de Biología Marina y Oceanografía 44(1): 257-262.

Chong J, C Oyarzún, E Aguilera \& A Arrizaga. 1991. Ciclo de maduración y puesta de la sardina española, Sardinops sagax (Jenyns 1842) (Clupeidae), en la zona pesquera de Talcahuano, Chile (1984-1985). Scientia Marina 55(2): $397-$ 404.

Claramunt G \& G Herrera. 1992. A new method to estimate the fraction of daily spawning females and the numbers of spawning in Sardinops sagax in northern Chile. Scientia Marina 58(3): 169-177.

Cubillos LA, DF Arcos, M Canales \& D Bucarey. 2001. Seasonal growth of small pelagic fish off Talcahuano $\left(37^{\circ} \mathrm{S}-\right.$ $73^{\circ} \mathrm{W}$ ): A consequence of their reproductive strategy to seasonal upwelling? Aquatic Living Resources 14: 115-124.

Cubillos L, M Canales, D Bucarey, A Rojas \& R Alarcón. 1999. Época reproductiva y talla de primera madurez sexual de Strangomera bentincki y Engraulis ringens en el período 1993-1997, en la zona centro-sur de Chile. Investigaciones Marinas 27: 73-85.

Elliott W, R Gonzáles, W Becerra \& A Ramírez. 2000. Biología y pesquería del pampanito Stromateus stellatus Cuvier, en la zona de Huacho (Perú) durante enero a septiembre 1999. Informes Progresivos, Instituto del Mar del Perú 112: 1-23.

Herrera G \& G Claramunt. 1990. Estimaciones de la fecundidad parcial y frecuencia de desove de Sardinops sagax durante 1987 y 1988, en el Norte de Chile. Revista de Investigaciones Científicas y Tecnológicas, Serie Ciencias del Mar 1: 55-68.
Hislop JRG, AP Robb \& JA Gauld. 1978. Observation on effects of feeding level on growth and reproduction in haddock, Melanogrammus aeglefinus (L.) in captivity. Journal of Fishery Biology 13: 85-98.

Hosmer D \& S Lemeshow. 1989. Applied logistic regression, 386 pp. John Wiley and Sons, New York.

Hunter JR \& SR Goldberg. 1980. The spawning incidence and batch fecundity in northern anchovy, Engraulis mordax. Fishery Bulletin 77: 641-652.

Hunter JR \& R Leong. 1981. The spawning energetics of female northern anchovy, Engraulis mordax. Fishery Bulletin 79: 215-230.

Leible M \& E Alveal. 1981. Catalogo de peces que habitan las aguas costeras y dulceacuícolas en la provincia de Concepción, 104 pp. Departamento de Biología y Tecnología del Mar, Pontificia Universidad Católica de Chile, Talcahuano.

Murúa H \& F Saborido-Rey. 2003. Female reproductive strategies of marine fish species of the North Atlantic. Journal of Northwest Atlantic Fishery Science 33: 23-31.

Murúa H, G Kraus, F Saborido-Rey, PR Witthames, A Thorsen \& S Junquera. 2003. Procedures to estimate fecundity of marine fish species in relation to their reproductive strategy. Journal of Northwest Atlantic Fishery Science 33: 33-54.

Oliva J, W Carvajal \& A Tresierra. 1986. Biología reproductiva e histología de gónadas en peces, 151 pp. Departamento de Ciencias Biológicas, Universidad Nacional de Trujillo, Trujillo.

Perrotta R, R Guerrero, C Carozza, P Quiroga \& G Macchi. 2006. Distribución y estructura de tallas de la palometa (Parona signata, Carangidae) y el pampanito (Stromateus brasiliensis, Stromateidae) en relación con las condiciones oceanográficas en la Zona Común de Pesca $\left(34^{\circ} \mathrm{S}-38^{\circ} \mathrm{S}\right)$ y estimación de la longitud de primera madurez sexual. Julio de 2001. Informe Técnico, INIDEP 75: 1-15.

Roa R, B Ernst \& F Tapia. 1999. Estimation of size at sexual maturity: an evaluation of analytical and resampling procedures. Fishery Bulletin 97(3): 570-580.

Sato T, HS Seo, Y Endo \& K Fujimoto. 2002. Diacyl glyceryl ether as the major muscle lipid in Stromateus stellatus and its hydrolyzability by lipase and oral acute toxicity on mice. Nippon Suisan Gakkaishi 68(4): 569-575.

SERNAPESCA. 2006. Anuario estadístico de pesca 2005, 210 pp. Servicio Nacional de Pesca, Ministerio de Economía, Fomento y Reconstrucción, Valparaíso.

Vizziano D \& N Borois. 1990. Ciclo histológico del ovario de Macrodon ancylon (Bloch \& Schneider 1801) (Teleostei: Scienidae). Biología Pesquera 19: 39-47.

Ware DM. 1984. Fitness of different reproductive strategies in teleost fishes. En: GW Potts \& RJ Wootton (eds). Fish reproduction strategies and tactics, pp. 349-366. Academic Press, New York.

Zar JH. 1984. Biostatistical analysis, 662 pp. Prentice-Hall, Englewood Cliffs. 